\title{
CONCEPCIONES EPISTEMOLÓGICAS DEL PROFESORADO DE BIOLOGÍA EN EJERCICIO SOBRE LA ENSEÑANZA DE LA BIOLOGÍA
}

\section{In-service Biology teachers Epistemological conceptions of Biology teaching}

\author{
Eduardo Ravanal Moreno ${ }^{1}$ • Mario Quintanilla Gatica ${ }^{2}$ • \\ Alberto Labarrere Surday ${ }^{3}$
}

Resumen: Debemos considerar importantes las concepciones del profesorado sobre la enseñanza de la biología y su relación con el desarrollo de competencias de pensamiento científico (CPC); para ello, hemos decidido identificar y caracterizar la noción enseñanza de la ciencia (EC) y cómo estas nociones inciden en el desarrollo de CPC, a partir de un cuestionario tipo Likert y un análisis de la información orientado desde la epistemología realista pragmática. Las representaciones docentes declaradas desde el cuestionario son complementadas a partir de textualidades docentes derivadas de entrevistas en profundidad, un grupo de discusión y un taller de reflexión docentes. Los principales resultados indican que la EC es preferentemente tradicional con una metodología de aprendizaje limitada a la transmisión de conocimiento enciclopédico matizada con una visión epistemológica tecnicista que no considera, en las racionalidades docentes, una enseñanza para el desarrollo de CPC.

Palabras clave: Enseñanza de biología. Enseñanza de la ciencia. Competencias. Pensamiento científico.

Abstract: We consider as important teacher's conceptions of teaching biology and their relation to the development of scientific thinking competencies (STC), so we have decided to identify and characterize notions of science education (SE) and how these affect the development of STC, We have departed from the application of a Likert type questionnaire and follow an analysis of the information from the viewpoint of a pragmatic realist epistemology. Teacher's reported representations derived from the questionnaire are supplemented with results of textual in-depth interviews, group discussion and a (theory-oriented) teacher's workshop. Main results indicate that Science Education is guided by a conception of a traditional learning methodology, which limits itself to the transmission of encyclopedic knowledge with a token of technological epistemology that it does not consider in the teaching rationales, teaching for the development of STC.

Keywords: Biology teaching. Science education. Scientific thinking. Competence.

\footnotetext{
${ }^{1}$ Escuela de Pedagogía en Biología y Ciencias, Facultad de Ciencias de la Educación, Universidad Central. Santa Isabel 1278, piso 6. Santiago, Chile.lravanalm@ucentral.cl

${ }^{2}$ Laboratorio de Investigación en Didáctica de las Ciencias, Pontificia Universidad Católica. Santiago, Chile.

${ }^{3}$ Escuela de Psicología, Universidad Santo Tomás. Santiago, Chile.
} 


\section{Introducción}

Estamos convencidos que la ciencia en la escuela debe permitir a los niños/as y jóvenes poder participar activamente de las cosas del mundo, debe ser, la ciencia en la escuela, un espacio diverso y rico de diálogos, debate, cuestionamiento y posibilidades de cambio y reestructuración de ideas como también surgimiento de otras nuevas. Desde esta perspectiva, la ciencia escolar se construye y concibe desde una imagen profundamente humana que rescata valores, vivencias, experiencias y situaciones contextuales y personales propias, rescata la cultura de un momento, de un tiempo o de una época con sus limitaciones y grandezas, como plantea Quintanilla (2006, p. 177): "La ciencia en la escuela un saber fascinante para aprender a leer el mundo", esto nos lleva a plantear una ciencia escolar con sentido y valor para todos los niños/as y jóvenes, que sea moderadamente racional; que permita a los estudiantes justificarla - ciencia escolar razonable - y de esa forma favorecer la participación activa para enfrentar nuevas interrogantes, nuevos desafíos, nuevas formas de mirar y enfrentar el mundo comprometidamente.

Por ello, la concepción sobre enseñanza de la ciencia declarada por el profesorado de ciencias debe virar hacia nuevos modelos teóricos que configuren una "nueva enseñanza de la ciencia en la escuela", en ese sentido, optamos por una ciencia escolar orientada hacia la formación de un sujeto competente en ciencias; que sabe, sabe hacer y actuar frente a las variadas situaciones que deberá enfrentar. Dichos propósitos, quizás, implícitos en el imaginario y acción profesional no son conscientes, lo que resta, a la hora de promover y regular la enseñanza para el aprendizaje. En esa dirección, creemos importante que la discusión con y entre profesores debe virar hacia la toma de consciencia del conocimiento sobre el conocimiento profesional y su regulación.

Estas primeras ideas sugieren un profesor o profesora altamente reflexivo, capaz de reconocer cómo aprenden los alumnos y conocedor de las teorías actuales sobre aprendizaje, es decir, un profesor que sabe - aprendió a - enseñar ciencias (ANGULO DELGADO, 2002). Ahora bien, un consideración importante es que los docentes no deben olvidar que los "conocimientos" no están "contenidos" en los libros, museos, CD o vídeo (IZQUIERDO, 2005) sino que surgen de actividades científicas escolares auténticas (RAVANAL; QUINTANILLA, 2008) que permiten al estudiantado participar, interrogar, juzgar, debatir, producir nuevo conocimiento en el contexto de la escuela, para lo cual, creemos necesario reorientar nuestros propósitos de enseñanza hacia el desarrollo de competencias de pensamiento científico (CPC).

En ese contexto, las CPC han de generar actividad científica escolar (ACE), a partir de situaciones en las que se pueda intervenir en el aula y fuera de ella con entidades y lenguajes apropiados para explicarlas y controlarlas teóricamente (QUINTANILLA, 2010). En este plano, se entiende que el desarrollo de CPC en un marco de actividad científica escolar permite que la experimentación, la modelización y la discusión se entrecrucen para promover una reconstrucción racional de los fenómenos del mundo (IZQUIERDO; SANMARTÍ; ESPINET, 1999). Sin embargo, la ciencia en la escuela, por las exigencias socio-culturales imperantes en el sistema de educación, puestas de manifiesto en largas discusiones sobre los puntajes del Sistema de Medición de la Calidad de la Enseñanza (SIMCE) o la Prueba de Selección Universitaria (PSU) obtenidos en cada establecimiento educacional, nos lleva a pensar que el énfasis radica en la enseñanza de los contenidos disciplinares, más que, discutir cómo los 
estudiantes se apropian de ellos, o, desde la "vereda del frente", de qué forma, el profesorado genera instancias de enseñanza-aprendizaje-evaluación que favorezcan la apropiación de conocimientos o, discutir qué consideraciones ha tomado para reestructurar ideas o teorías implícitas en cada estudiantes desde la noción de competencia de pensamiento científico que comparte y declara desde su actuación pedagógica.

Esto nos obliga a reconocer que la enseñanza de las ciencias en la escuela debe superar la visión reducida de qué, en la escuela, se aprende biología, química o física, cuando los estudiantes son "capaces" - temporalmente - de resolver un ejercicio, mencionar atributos o propiedades físico-químicas de hechos o fenómenos biológicos o, cuándo repiten, inequívocamente, un listado de estructuras del cuerpo humano. La ciencia en la escuela debe superar lo netamente instrumental y debe generar un espacio que permita a todos los estudiantes encontrar una nueva forma de mirar el mundo (ALIBERAS, 2005); de manera que permita la participación en las decisiones socio científicas propias de una sociedad en permanente cambio (QUINTANILLA, 2007).

La imagen de ciencia, especialmente la tendencia epistemológica de la ciencia escolar y su enseñanza-aprendizaje-evaluación en el aula nos parece interesante de conocer y discutir, dado que, estas tendencias epistémicas de ciencia que existen en los docentes no se transparentan durante la clase de ciencias, lo que trae como consecuencias, favorecer en niños y jóvenes una imagen de ciencia racionalmente fuerte, rígida, poco flexible. Por lo anterior el propósito de este artículo es identificar y caracterizar las concepciones del profesorado de biología en ejercicio sobre la enseñanza de las ciencias y la(s) implicancia(s) de estas en el desarrollo de CPC en la escuela secundaria.

\section{Enseñanza de la biología}

Desde finales de la década de los noventa, muchos de los esfuerzos por el mejoramiento de la calidad de la educación científica se han centrado en la exploración de las ideas de los y las estudiantes frente a la ciencia y a los conceptos científicos que se enseñan en los diferentes niveles educativos. Izquierdo (2000) y Adúriz-Bravo (2001) intentan establecer una base epistemológica para la enseñanza de las ciencias a la luz de las nociones contemporáneas sobre la naturaleza de la ciencia y de cómo aprenden los niños y niñas, adolescentes y jóvenes, lo cual constituye un aporte muy valioso a la reforma curricular, que se plantea desde perspectivas diferentes, pero complementarias: filosofía e historia de la ciencia, psicología del aprendizaje y didáctica de las ciencias. Sin embargo, un hecho evidente es que cualquier innovación educativa debe ser a partir de la formación misma del profesorado de ciencias, o por lo menos tener en cuenta la preparación profesional del docente. La enseñanza de las ciencias y su aprendizaje en la escuela, en un marco de actividad científica escolar auténtica (RAVANAL; QUINTANILLA, 2008), exige tomar ciertos acuerdos vinculados con la concepción de ciencia que comparte el profesorado, dado que, asuntos relativos a qué es la ciencia, cómo y qué implica su construcción; son aspectos importantes en la alfabetización científica y tecnológica de las personas (ACEVEDO, 2007). Desde esta perspectiva, la naturaleza de la biología en el contexto escolar primario y secundario implica conocimiento metacientífico con valor para la educación en ciencia con la finalidad de promover reflexión, posición epistemológica y mejorar la ense- 
ñanza y aprendizaje de los contenidos científicos (ADÚRIZ-BRAVO, 2007). Creemos que la resignificación sobre la naturaleza de la ciencia y, particularmente de la biología escolar, en el profesorado contribuye a la enseñanza y aprendizaje de la ciencia escolar, en la medida que, favorezca espacios de participación dialógicos para una construcción racional y razonables (IZQUIERDO, 2007) contrarias a ideas de un conocimiento acabado y verdadero existente en muchos profesores (SETTLE, 1990). Un estudio realizado por Flores et al. (2007) reveló que la tendencia epistemológica de profesores de biología de secundaria se encuentra "cristalizada" en el positivismo lógico caracterizada por una noción de conocimiento objetivo y acumulativo al igual como lo planteó Settle (1990). Al respecto, resultan interesantes los aportes de Carvajal y Gómez (2002) si consideramos la finalidad intrínseca-cultural y valórica de la naturaleza de la ciencia, en estos contextos, y de sus finalidades; pensando en la necesidad de un nuevo profesor/a de ciencia para una nueva sociedad que nos demanda nuevos compromisos de futuro. Estas autoras, luego de analizar las concepciones de ciencia y aprendizaje de profesores de educación secundaria y bachillerato en México, revelan la mermada capacidad reflexiva sobre aspectos culturales, éticos y filosóficos de la ciencia. En esa dirección, no se puede enseñar aquello que no se conoce, y sobre todo, sino se ha reflexionado suficientemente sobren ello (GUISÁSOLA; MORENTIN, 2007). Esto nos lleva a plantear que los docentes no pueden abordar la enseñanza hacia la construcción de conocimiento independiente del análisis preliminar de la ciencia planificada y propuesta en el aula, dado que, el sistemas de ideas que configuran la epistemología del profesor emerge diferenciadamente en distintos contextos; asunto que nos lleva describir un docente teóricamente tradicional-dogmático que dé cuenta de una concepción de aprendizaje "constructivista” como lo plantea Mellado (1996).

En un estudio realizado por Ravanal (2010) se evidenció que los profesores de biología manifiestan una visión empírica de la ciencia, no alejada de otras concepciones docentes, en que la diversidad epistemológica guarda relación con el racionalismo, relativismo y empirismo (MARTÍNEZ; MARTÍN DEL POZO; RODRIGO, 2001) en la que el empirismo es la más frecuente en docentes de ciencias primaria activo y de magisterio (PORLÁN, 1989 apud RUIZ et al., 2005). Martínez, Martín del Pozo y Rodrigo (2001) sostienen que de la diversidad de concepciones existente en profesores, el conocimiento científico para ellos es visto como superior, objetivo, neutral y descontextualizado, aspecto relacionado con algunas ideas persistentes en los profesores analizados, lo que nos lleva plantear nuevas formas de abordar o reestructurar el diseño de discusión sobre la enseñanza de la biología; naturaleza de la ciencia - biología.

Considerando estos aspectos, nos parecen interesantes recoger los factores específicos que dificultan una enseñanza más adecuada de la naturaleza de la ciencia propuestos por Kim et al. (2005 apud ACEVEDO, 2007) quien plantea que las dificultades pasan por que existe:

a) Confusión entre naturaleza de la ciencia y procesos de la ciencia: Una enseñanza basada en los procedimientos científicos no implica necesariamente una buena comprensión de la naturaleza de la ciencia (EICK, 2000).

b) Desconocimiento de enfoques didácticos eficaces para la enseñanza de la biología: Derivado del escaso dominio del profesorado a la hora de evidenciar explícita y reflexiva la enseñanza de la naturaleza de la ciencia - biología (ACEVEDO, 2007). 
c) Falta de conocimiento profundo del tema científico. El profesorado debe propiciar para una buena comprensión de los conceptos científicos vínculos entre ciencia, ciudadanía y valores (QUINTANILLA, 2006a).

\title{
Competencias de pensamiento científico: una finalidad de la enseñanza de la biología
}

Para iniciar la discusión deseamos compartir la definición de competencia propuesta por Chamizo e Izquierdo (2007, p. 13) quienes afirman que:

\begin{abstract}
Brevemente se puede decir que una competencia apela a saber, a saber hacer, a ser, a vivir con otros en situaciones de la vida en las cuales se ha de decidir cómo actuar. Si la competencia tiene que ver con la ciencia, la situación es tal que moviliza conocimientos que no pueden ser «de libro», sino que corresponden a una actividad científica. Así, no se puede aislar el saber científico de la vida: de sus aplicaciones, de sus implicaciones, de su significado en relación a otras materias.
\end{abstract}

Para Camacho y Quintanilla (2008, p. 198) las competencias científicas son complejas, dinámicas y difusas que se entienden como: "habilidades y destrezas que permitan a los estudiantes mediante procesos reflexivos la construcción y resignificación de los conocimientos científicos que aprenden".

En ese sentido, las competencias, plantea Quintanilla (2006b), en el campo de la educación científica, se corresponden con la capacidad de las personas para afrontar situaciones nuevas a partir de los conocimientos aprendidos; lo que se contempla tanto en el ámbito cognitivo, valórico y cultural y, tal como lo señala Labarrere (2008), depende del sujeto y de su contexto. Camacho y Quintanilla (2008) afirman que estas competencias deben estar vinculadas con la teoría científica en discusión en el aula, dado que estas permiten articular el pensamiento, el lenguaje y la acción en los sujetos, para dar cuenta de los nuevos conocimientos científicos construidos paulatinamente. La enseñanza para el desarrollo de CPC no significa capacidad de un sujeto para resolver un problema que ya viene formulado en términos disciplinares, sino hacer uso de manera autónoma de los conocimientos científicos construidos para identificarlos en situaciones reales (GONZÁLEZ et al., 2005). Ahora bien, para la Organización para la Cooperación y el Desarrollo Económico (2008) en su prueba del Programa para la Evaluación Internacional de Alumnos (PISA) 2006 incorpora la evaluación en torno a las competencias científicas y para ello asume que una competencia científica en referencia a las siguientes habilidades del sujeto:

. Conocimiento científico y utilización de ese conocimiento para identificar cuestiones, adquirir nuevos conocimientos, explicar fenómenos científicos y sacar conclusiones a partir de pruebas en problemas relacionados con las ciencias. Por ejemplo, cuando los individuos leen sobre una 
Ravanal, E. M.; Quintanilla, M. G.; Labarrere, A. S.

cuestión relacionada con la salud, ¿pueden separar los aspectos científicos del texto de los no científicos y pueden aplicar sus conocimientos y justificar decisiones personales?

- Comprensión de los rasgos característicos de las ciencias como forma humana de conocimiento e investigación. Por ejemplo, ¿conocen los individuos la diferencia entre las explicaciones basadas en pruebas y las opiniones personales?

- Conciencia de cómo las ciencias y la tecnología dan forma a nuestros entornos materiales, intelectuales y culturales. Por ejemplo, ¿pueden los individuos reconocer y explicar la influencia que tienen las tecnologías en la economía, la organización social y la cultura de una nación? ¿Son conscientes los individuos de los cambios medioambientales y de los efectos que tienen dichos cambios en la estabilidad económica y social? . Voluntad de involucrarse como ciudadano reflexivo en cuestiones relacionadas con las ciencias y con las ideas científicas. Esto se refiere al valor que los alumnos otorgan a las ciencias, tanto en los temas como en el enfoque científico para comprender el mundo y resolver problemas. [...]. (ORGANIZACIÓN PARA LA COOPERACIÓN Y EL DESARROLLO ECONÓMICO, 2008, p. 39)

Estas habilidades propias del sujeto para adquirir ciertas competencias científicas propuestas por PISA 2006 demandan un cambio en la forma de abordar los conocimientos científicos en el aula; la clase de biología exige espacios de debate que promueva el desarrollo de competencias; desde esta perspectiva, entendida como una elaboración personal-social que adopta una configuración individual y personalizado, que se expresa de manera peculiar en cada persona (LABARERRE, 2008); aspecto de alta complejidad que dista de la propuesta de competencia de PISA 2006. Por su parte, Quintanilla (2012) señala que la competencia es la capacidad de responder con éxito a las exigencias personales y sociales que nos plantea una actividad científica en el contexto del ejercicio profesional e implica dimensiones de tipo cognitivo como no cognitivo, esto justifica nuestra idea de caracterizar ámbitos de una competencia, dado que, lo que existe no es la competencia en sí misma, sino un sujeto competente, por ello, Quintanilla (2012) afirma que la competencia es un tipo de conocimiento complejo que siempre se ejerce en un contexto de manera eficiente, que exige una combinación de aptitudes prácticas y cognitivas, de orden diverso que conjuntamente ponen en funcionamiento la realización eficaz de una acción. Suma a esta noción de competencia las ideas de Labarrere (2008, p. 3) quien plantea: "concebimos la competencia más allá de un mero "ajuste” a determinadas condiciones y exigencias "fuera" del sujeto y, sin dejar de considerar que las demandas del contexto, las condiciones, otras, son básicas".

Desde esta perspectiva de análisis, creemos que una competencia de pensamiento científico involucra procesos de pensamiento propios de un sujeto que al enfrentar una situación involucra conocimiento, procedimientos, técnica, valores, motivaciones personales, historia y experiencia. Por todo lo anterior, creemos que el diseño de la enseñanza debe orientarse desde y hacia el desarrollo de competencias científicas como consecuencia de una actividad cognitiva que moviliza al sujeto en todos los planos de desarrollo (LABARRERE; QUINTA- 
NILLA, 2002), desde ahí, pareciera pertinente, hablar de competencias de pensamiento científico; en ese sentido Labarrere (2012, p. 73) específica que éstas deben ser entendidas como "una formación personal referida al desempeño del sujeto en contextos de actuación que demandan pensamiento y la acción".

Convencidos de que el desafío pasa por, "pensar" la enseñanza para la formación de un sujeto competente, tanto, individual como colectivamente, que sabe cómo y cuándo hacer uso del conocimiento considerando contextos, situaciones, formas de hacer y decir, entre otras, deseamos dejar planteado que una Competencia de Pensamiento Científico: se concibe como un proceso de desarrollo sistemático y continuo donde se articulan diferentes planos de análisis que movilizan valores, actitudes, procedimientos, habilidades y emociones, de manera consciente e intencionada y en la cual se concibe el conocimiento científico como una actividad humana cuyas finalidades articulan las teorías (racionalidad moderada) con el mundo (razonabilidad compartida). Ese sería por así decirlo, nuestro sujeto competente. Idea que ya se viene trabajando desde el año 2007 con los proyectos del Fondo Nacional de Desarrollo Científico y Tecnológico (FONDECYT) 1070795 (QUINTANILLA et al., 2007), 1095149 (QUINTANILLA et al., 2009), 1110598 (QUINTANILLA et al., 2011), los cuales han sugerido diferentes directrices teóricas y metodológicas al respecto de la formación de docentes y la enseñanza de las Ciencias en Chile.

\section{Contexto de la investigación}

En el marco del proyecto Fondecyt 1070795 (QUINTANILLA et al., 2007) se realiza una convocatoria a profesores de ciencias naturales: biología, química y física para realizar un taller de formación sobre enseñanza, aprendizaje y evaluación de aprendizajes en ciencia. En la primera sesión de trabajo, se aplica un cuestionario tipo Likert con 80 afirmaciones para 8 dimensiones de análisis. El propósito es describir y caracterizar la imagen de ciencia en el profesorado de ciencia en torno a las siguientes dimensiones metateóricas: Naturaleza de las ciencias, enseñanza de las ciencias, aprendizaje de las ciencias, evaluación de los aprendizajes científicos, rol del profesor, historia de las ciencia, resolución de problemas y competencias de pensamiento científico. Se proponen 10 enunciados por dimensión metateórica que representan dos visiones epistemológicas antagónicas: tradicional-dogmática y constructivista. En esta comunicación sólo se aborda la dimensión Enseñanza de la Ciencia.

\section{Muestras y técnicas}

Un total de 53 profesores de biología responde el cuestionario. De los 53 docentes, cinco (5) pertenecen a colegios particulares pagado de Santiago y 48 se desempeñan como profesores en colegios particulares subvencionado (22), o de carácter municipalizado (26). Cuatro (4) profesores en un colegio particular subvencionado y municipalizado, otros dos (2) docentes lo hacen en ambos tipos de establecimientos. De los 53 profesores, 22 trabajan con estudiantes de primer a cuarto año medio, la diferencia se distribuyen entre los niveles mencionados con un promedio de 31 profesores. 
La primera etapa del análisis surge de la información colectada a partir del cuestionario tipo Likert. La información recogida fue tabulada para luego realizar un análisis estadístico descriptivo con el propósito de explorar las nociones sobre la imagen de ciencia-biología de los docentes. La información nos permite proponer una descripción e interpretación según las dimensiones de análisis propuestas pon el cuestionario como una mirada global de ciertas nociones que eventualmente pueden estar explicitadas al momento de abordar el cuestionario. El enfoque epistemológico con el cual se interpretan las adhesiones o no a los enunciados del cuestionario es realista pragmática.

\section{Cuestionario tipo Likert}

Con el propósito de identificar y caracterizar las nociones del profesorado de biología en activo sobre enseñanza de la ciencia, se rediseña un instrumento tipo Likert propuesto por Quintanilla et al. (2006) diseñado para develar las concepciones metateóricas sobre naturaleza de ciencia y su enseñanza en profesores de ciencias en ejercicio de enseñanza media, así como las consecuencias que ello tiene para el aprendizaje. El instrumento original está constituido por seis (6) dimensiones de análisis, el rediseño se centra en definir, diseñar y validar dos nuevas dimensiones de análisis, competencia de pensamiento científico y resolución de problemas. En el mismo instrumento, se recogieron datos de edad de los docentes; dependencia administrativa del colegio; nivel en que desarrolla sus clases; género; años de experiencia profesional y universidad de procedencia, ya que como afirma Lederman (1999) los contextos son sumamente importante en cualquier investigación sobre las prácticas y creencias de los profesores.

\section{Elaboración de enunciados para diseño del cuestionario}

Se consulta bibliografía con el propósito de proponer enunciados preliminares que fueron caracterizadas y disgregadas metodológicamente en el cuestionario, pertinentes desde el enfoque que delimita nuestros ejes teóricos. Para la propuesta de enunciados (E) se considera los elementos del contrato didáctico, de esa forma existen enunciados orientados hacia el profesor, estudiante y el saber erudito; cada uno de ellos representativo de una visión epistemológica particular: tradicional-dogmática o constructivista. Los enunciados propuestos para la noción epistemológica tradicional, están situadas en la visión positivista y/o empirista; como también, enunciados que atienden a la visión constructivista evolutiva-realista pragmática de Toulmin (1977) y Giere (1992). Los enunciados se presentan en el cuadro 1 del acápite resultados.

\section{Enunciados para la dimensión Enseñanza de la Ciencia}

\section{Validación del cuestionario}

Se ha intentado que cada uno de los ítems que componen el instrumento evalúe efectivamente las categorías predeterminadas, y a su vez, que recojan de forma amplia el pensamiento de los profesores de biología con respecto a ellas, de manera tal que este se convierta en una fuente de información importante para la transformación y consolidación de 
las prácticas de enseñanza de las ciencias, hacia la formación de sujetos competentes en ciencia (IZQUIERDO, 2007), para ello, el diseño de la enseñanza debe estar centrado en el desarrollo del pensamiento más que en la acumulación de contenidos carentes de significado y valor para muchos estudiantes. Por ello, es importante conocer las concepciones implícitas y explícitas en los profesores de biología de y sobre la ciencia que enseñan.

En una primera instancia, el instrumento fue sometido a un proceso de determinación de su validez interna, por parte de especialistas en el área de metodología e investigación en didáctica de las ciencias, y a un grupo piloto de 20 profesores de ciencias naturales en ejercicio, en la ciudad de Santiago, que ejercen en colegios municipalizados y particulares pagados.

\section{Análisis de datos}

Con el propósito de identificar, caracterizar e interpretar tendencias epistemológicas presentes en el profesorado se realiza un análisis que considera tres etapas:

1) Descripción e interpretación a partir de un análisis estadístico descriptivo. A pesar que la técnica se enmarca en la metodología cuantitativa, el análisis que proponemos, es más bien de carácter cualitativo, dado que, intenta describir o interpretar la tendencia epistemológica del docente, representada por el mayor o menor grado de adhesión a un enunciado, expresado en el porcentaje obtenido de la suma de las opciones Totalmente de acuerdo y Parcialmente de acuerdo. Junto con esto, proponer categorías de análisis que nos permitan caracterizar las tendencias epistemológicas de los docentes de biología.

2) Descripción e interpretación desde un análisis de componentes principales. El propósito es explorar e identificar aquellos enunciados que teóricamente representan las concepciones del grupo de profesores encuestados. En ese sentido, el análisis factorial nos permite reducir la información y constituir grupos que permitan tipificar la tendencia epistemológica del profesor de biología en un momento particular.

3) Categorizaciones de las concepciones docentes. Para poder categorizar las concepciones del profesorado, se utiliza una red conceptual propuesta por Wang y Marsh (2002) para la historia de la ciencia que considera tres niveles de comprensión docente: conceptual, contextual y procedimental. Dichos niveles nos permiten enriquecer el perfil epistemológico del profesor y desde ahí la concepción que comparte.

Los insumos de información para los análisis propuestos surgieron del cuestionario tipo Likert y de otros contemplados en el marco de la investigación, tales como: grupo de discusión (GD), entrevista en profundidad (EP), taller de reflexión docente (TRD), análisis de producciones docentes y observaciones de clase.

\section{Resultados}

Parte I.

Análisis estadístico descriptivo para la dimensión Enseñanza de las Ciencias

A continuación los resultados obtenidos para la dimensión Enseñanza de la ciencia en discusión (Tabla 1). 
El análisis para esta dimensión nos permite afirmar que los docentes creen que los modelos teóricos en discusión se justifican a través de la experimentación (E64), esto nos lleva a pensar en una epistemología empiricista que pretende que exista una conexión entre los términos teóricos y los términos experimentales. Esta visión restringe en los estudiantes la oportunidad de introducir juicios de valor, además se plantea una imagen de la biología no evolutiva (E63). Sin embargo, los docentes asumen teóricamente una enseñanza del método científico reflexivo (E59) aspecto que desde esta perspectiva es racional y moderada, siempre que la finalidad de enseñarlo se vincule con el mundo real de los estudiantes, que aparentemente no son las teorías discutidas en la sala de clase ni menos los experimentos diseñados para la verificación y justificación de estos.

Tabla 1. Medidas de tendencia central y porcentajes para las opciones Totalmente de acuerdo y Parcialmente de acuerdo según 53 profesores de biología en activo.

\begin{tabular}{|c|c|c|c|c|c|c|c|c|c|c|}
\hline \multirow{2}{*}{$\begin{array}{c}\text { Medidas de tendencia central } \\
\text { y dispersión }\end{array}$} & \multicolumn{5}{|c|}{ Tradicional-dogmático } & \multicolumn{5}{|c|}{ Constructivista } \\
\hline & E46 & E72 & E63 & E64 & E71 & E59 & E18 & E28 & E2 & E21 \\
\hline Media & 2,08 & 2,86 & 1,48 & 1,82 & 3,09 & 3,67 & 3,79 & 3,43 & 3,65 & 3,54 \\
\hline Desviación estándar & 0,85 & 0,98 & 0,56 & 0,81 & 0,84 & 0,57 & 0,48 & 0,87 & 0,54 & 0,53 \\
\hline $\begin{array}{l}\text { Porcentaje (Totalmente } \\
\text { y Parcialmente de acuerdo) }\end{array}$ & 77,5 & 37,5 & 92,5 & 87,5 & 82,5 & 92,5 & 97,5 & 90,0 & 97,5 & 97,5 \\
\hline
\end{tabular}

Fuente: Elaborado por los autores

Creemos que los docentes se sitúan en dos espectro de análisis de la realidad para un estudiante: "El mundo de las teorías" que debe aprender para rendir pruebas externas o internas, como lo muestra los datos obtenidos para E2 y, "el mundo socio cultural" propio del alumno en donde la biología es un valioso aporte siempre que las temáticas se correspondan con estos aspectos, que pueden estar representados por los enunciados 18 y 21. Estos antecedentes son enriquecidos con algunas textualidades derivadas de entrevistas en profundidad sobre qué es ciencia y su enseñanza; del taller de reflexión docente sobre el diseño de la enseñanza para la noción de metabolismo en estudiante de primer año medio y un grupo de discusión sobre ciencia y competencias de pensamiento científico. Algunas textualidades de interés son:

\footnotetext{
"La ciencia (biología) debería ser enseñada desde los chicos, ¿Cierto? $[\ldots]$ Que tengan cierto manejo para enfrentarse a lo cotidiano [...] Sin embargo, en los colegios estamos sometidos a presiones que nos alejan abismantemente de ese camino como por ejemplo rendir la PSU, rendir un Simce en la cual tenemos que mecanizar a los estudiantes... en post de un beneficio a corto plazo [...]" . (Profesora 1. GD)

"[...] Yo la veo (biología) desde ese punto de vista del autocuidado de ellos". (Profesora 2. EP)
} 
Luego de analizar un conjunto de actividades vinculadas con la enseñanza del metabolismo en el TRD, las profesoras participantes sostienen que un estudiante "Debe saber los componentes de cada una de las macromoléculas. Tener claro la función de la mitocondria y finalidades de las reacciones químicas" (Profesora 3. TRD-03). Si revisamos los enunciados E2, 18, 21, 28 y 59 (Cuadro 1) todos representan a la visión epistemológica constructivista, a la cual adhieren mayoritariamente los docentes encuestados, lo que nos lleva a pensar que la coexistencias teórica existente en ellos, las orientaciones ministeriales y el énfasis en una educación de calidad medida desde los productos, junto con las exigencias sociales, provocan en los docentes confusión y apego hacia tendencias epistemológicas tradicionales que restringen "las buenas intenciones constructivistas" declaradas en el cuestionario (Gráficos 1 y 2).

Ahora bien, desde las visiones epistemológicas en estudio podemos afirmar que existe una tendencia constructivista hacia las finalidades implícitas en la enseñanza de la biología, como cambios en la forma de actuar, dado que, se han instalados modos o protocolos de acción, que responden a competencias de pensamiento científico.

Por otra parte, las intenciones constructivista declaradas desde las finalidades de la enseñanza de la biología se cruzan con nociones de aprendizajes propios de visiones epistemológicas conservadoras o tecnológicas, en la que percibimos que el aprendizaje es entendido más bien por sustitución que por evolución o resignificación de ideas vinculadas a una noción

Cuadro 1. Enunciados relacionados con la dimensión enseñanza de las ciencias

\begin{tabular}{|c|c|}
\hline \multicolumn{2}{|r|}{ Dimensión Enseñanza de las Ciencias - Biología } \\
\hline Enunciados & Ítems específicos para la dimensión \\
\hline E2 & $\begin{array}{l}\text { La enseñanza de teorías científicas debe promover la relación entre los conceptos } \\
\text { científicos, en los diferentes campos de un saber erudito. }\end{array}$ \\
\hline E18 & $\begin{array}{l}\text { La enseñanza de las ciencias promueve en el estudiantado, una actitud ciudadana crítica y } \\
\text { responsable }\end{array}$ \\
\hline E21 & La enseñanza de las ciencias permite explicar el mundo cotidiano con teoría científica. \\
\hline E28 & $\begin{array}{l}\text { La enseñanza de las ciencias en el aula debe considerar el significado que los estudiantes } \\
\text { tienen de un concepto, aunque éste no corresponda con el significado científico correcto. }\end{array}$ \\
\hline E59 & $\begin{array}{l}\text { La enseñanza reflexiva del método científico permite que el estudiantado cambie su forma } \\
\text { de actuar frente a nuevas situaciones del mundo real. }\end{array}$ \\
\hline E46 & $\begin{array}{l}\text { La enseñanza de las ciencias se basa en dejar que los estudiantes descubran, por sí } \\
\text { mismos, los conceptos científicos. }\end{array}$ \\
\hline E63 & $\begin{array}{l}\text { La enseñanza de las ciencias permite que los estudiantes reemplacen sus modelos } \\
\text { incorrectos acerca de la realidad, por conceptos científicamente correctos. }\end{array}$ \\
\hline E64 & $\begin{array}{l}\text { Las actividades experimentales son imprescindibles para justificar la enseñanza de los } \\
\text { modelos teóricos. }\end{array}$ \\
\hline $\mathrm{E} 71$ & $\begin{array}{l}\text { En la enseñanza de las ciencias se obtienen aprendizajes definitivos, aún si no se } \\
\text { consideran los conocimientos previos }\end{array}$ \\
\hline $\mathrm{E} 72$ & $\begin{array}{l}\text { La ciencia que se enseña en el aula es un conocimiento sin componentes ideológicos, } \\
\text { sociales y culturales. }\end{array}$ \\
\hline
\end{tabular}

Fuente: Elaborado por los autores 
Ravanal, E. M.; Quintanilla, M. G.; Labarrere, A. S.

Gráfico 1. Concepciones de los docentes de biología sobre Enseñanza de la Ciencia (EC) según los enunciados propuestos en el cuestionario tipo Likert. Se grafican los enunciados en que los docentes están total o parcialmente de acuerdo con la afirmación propuesta.

72. La ciencia que se enseña en el aula es un conocimiento sin componentes ideológicos, sociales y culturales

71. En la enseñanza de las ciencias se obtienen aprendizajes definitivos ...

64. Las actividades experimentales son imprescindibles para justificar la enseñanza

63. La enseñanza de las ciencias permite que los estudiantes reemplacen sus modelos incorrectos ...

46. La enseñanza de las ciencias se basa en dejar que los estudianes descubran ...

59. La enseñanza reflexiva del método científico permite que el estudiantado cambie su forma de actuar ...

28. La enseñanza de las ciencias en el aula debe considerar el significado que los estudiantes ...

21. La enseñanza de las ciencias permite explicar el mundo cotidiano con teoría científica

18. La enseñanza de las ciencias promueve en el estudiantado, una actitud ciudadana crítica y responsable

2. La enseñanza de teorías científicas debe promover la relación entre los conceptos científicos ...

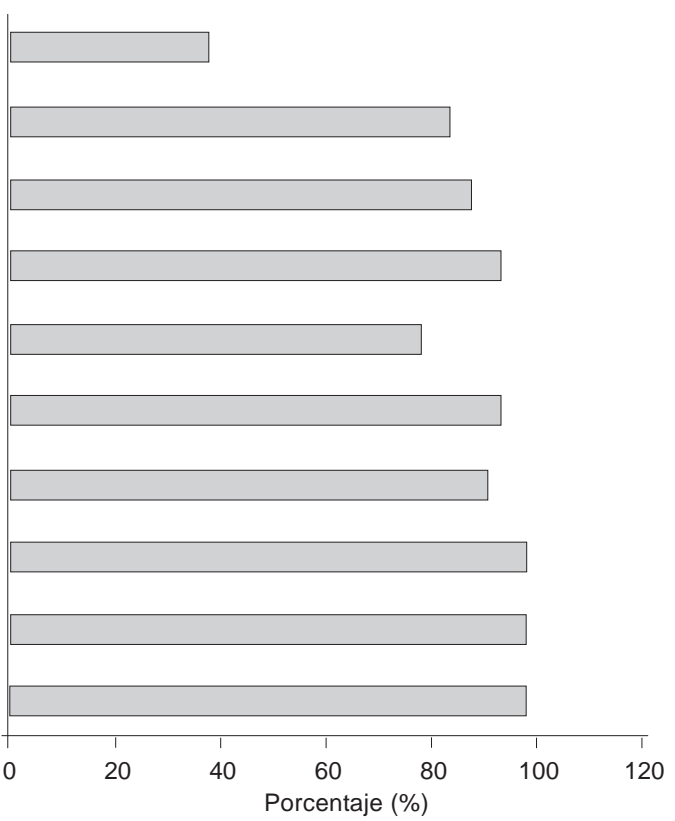

Fuente: Elaborado por los autores

Gráfico 2. Tendencias epistemológicas sobre la enseñanza de la biología en el profesorado en ejercicio.

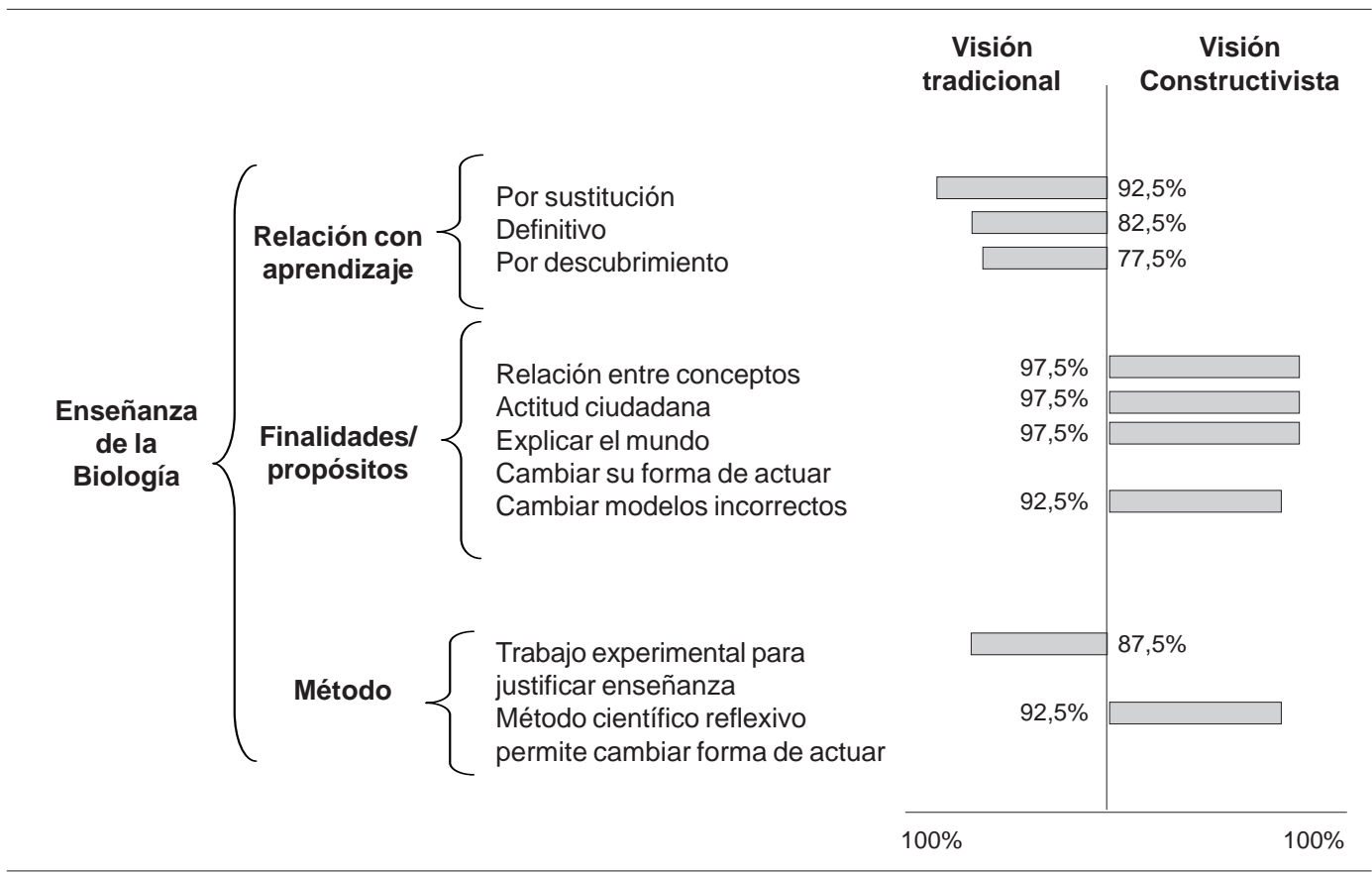

Fuente: Elaborado por los autores 
científica. En relación a las maneras de abordar la enseñanza de la biología, se aprecia valoración por los espacios de razonamiento hipotéticos-deductivos reflexivos, que resultan contrarios a esperar que estos espacios de razonamiento se conciban como "herramientas" de justificación. Esto último aún persistente en el profesorado de biología encuestado.

\title{
Parte II.
}

\section{Red conceptual para la categorización de las concepciones docentes} para la dimensión Enseñanza de la Ciencia - Biología

Considerando los análisis anteriores, podemos evidenciar que los docentes de biología sitúan sus racionalidades, al referirse a la enseñanza de la ciencia, en ámbitos de comprensión conceptual y contextual (Cuadro 2). Surge una noción de un contenido científico absoluto, caracterizado por una construcción lógica incuestionable, lo que hace de la enseñanza de la biología pobremente racionaly razonable. Creemos que la enseñanza de la ciencia (biología) no se construye en la escuela en un marco de actividad científica escolar desde la concepción evolutiva de las ideas o modelos teórico propios de los sujetos que aprenden. Algunas textualidades interesantes que deseamos compartir y que permiten de algún modo delimitar el análisis son:

\begin{abstract}
"Que el niño sea capaz de adquirir aprendizajes significativos con esa ciencia, no sea tanto la cantidad, porque el ministerio da una cantidad estratosférica como contenidos, sino que sea capaz de que lo que yo le estoy entregando [...] de adquirir cambios de conductas, aprendizaje significativo [...] Obviamente la biología es que aprendan, para que adquieran aprendizaje significativo, que sean capaces de si uno les hace una pregunta de aplicación, de enfrentarse, de contestar esa pregunta, eso es para mí los cambios de conductas, es lo principal”. (Profesora 2; EP)

“[...] enseñar a construir su conocimiento, entreguemos, entreguemos algunas cosas, y es lo que se intenta probablemente pero hay problemas de tiempo, definitivamente, así por ejemplo, ser más práctico, hacer laboratorio, hagamos esto así, pero hagámoslo prácticamente, cues-
\end{abstract}

Cuadro 2. Red conceptual para la dimensión Enseñanza de la Ciencia (EC)

\begin{tabular}{|c|c|}
\hline $\begin{array}{c}\text { Comprensión } \\
\text { conceptual }\end{array}$ & $\begin{array}{c}\text { Proporciona contrastación, comparación de ideas para la formación o reestructuración de } \\
\text { un modelo teórico que permita predecir, deducir, descubrir para aprender con sentido. }\end{array}$ \\
\hline $\begin{array}{c}\text { Comprensión } \\
\text { contextual }\end{array}$ & $\begin{array}{l}\text { La enseñanza vinculada a un componente ideológico, social y cultural } \\
\text { La enseñanza como promotor de un ciudadano crítico y responsable }\end{array}$ \\
\hline $\begin{array}{c}\text { Comprensión } \\
\text { procedimental }\end{array}$ & $\begin{array}{l}\text { La enseñanza de la ciencia escolar desde: } \\
\text {. La experimentación } \\
\text {. El método científico }\end{array}$ \\
\hline
\end{tabular}

Fuente: Elaborado por los autores 
Ravanal, E. M.; Quintanilla, M. G.; Labarrere, A. S.

ta, cuesta entregarles todas las herramientas a los chicos para que se despierten o para que construyan su conocimiento de a poco, la experiencia me dice que no." (Profesor 4, GD)

\section{Parte III.}

Análisis de componente principales (ACP) para la dimensión Enseñanza de las Ciencias (EC)

El ACP para la dimensión EC se realizó con un coeficiente de correlación superior a 0,5. Los enunciados (E) utilizados para el análisis y que corresponden a la dimensión de interés se indican en el Cuadro 3 además del valor de correlación para el primer factor del ACP.

Cuadro 3. Correlación por enunciado para el Factor 1 del análisis de componente principal para la dimensión Enseñanza de la Ciencia - Biología

\begin{tabular}{|c|c|c|c|}
\hline Enunciado & $\begin{array}{c}\text { Noción epistemológica según } \\
\text { cuestionario tipo Likert }\end{array}$ & $\begin{array}{c}\text { Elemento del contrato didáctico } \\
\text { que representa el enunciado }\end{array}$ & Correlación \\
\hline E64 & Tradicional-dogmática & Profesor & 0.806 \\
\hline E63 & Tradicional-dogmática & Profesor & 0.684 \\
\hline E46 & Tradicional-dogmática & Profesor & 0.566 \\
\hline E71 & Tradicional-dogmática & Profesor & 0.394 \\
\hline E72 & Tradicional-dogmática & Profesor & 0.054 \\
\hline E28 & Constructivista & Profesor & -0.045 \\
\hline E2 & Constructivista & Profesor & -0.223 \\
\hline E18 & Constructivista & Profesor & -0.370 \\
\hline E59 & Constructivista & Profesor & -0.416 \\
\hline
\end{tabular}

Fuente: Elaborado por los autores

El Factor 1 del ACP para la dimensión Enseñanza de las ciencias, revela la tendencia de los docentes hacia la visión epistemológica tradicional-dogmática en la que los enunciados E64, E63, E46 y E71 tienen el más alto valor de correlación, esto nos lleva a afirmar que los docentes creen que la enseñanza de la ciencia - biología - permite lograr aprendizajes definitivos a pesar de no considerar los conocimientos previos, emerge la noción de que las ideas y la evolución de ellas no son relevantes durante la enseñanza (E71 y E63), al parecer los contenidos disciplinares son importantes en si mismo, aspecto que adquiere valor cuando los profesores reconocen que la experimentación permite justificar esos contenidos (E64) (Figura 1).

Considerando los aportes de Porlán y Martín del Pozo (2004) la enseñanza es dogmática y tradicional con una metodología de aprendizaje limitada a la transmisión de conocimiento enciclopédico que se matiza con una epistemología tecnicista que da cuenta de un enfoque empiricista (E64) con una metodología basada en los pasos del método científico para verificar la teoría que se ha discutido o bien una enseñanza de la ciencia basada en el descubrimiento, por si mismo, de los conceptos científicos (E46). Insistimos que las tendencias dogmáticas sobre la enseñanza de la biología del profesorado radican principalmente por: 
Figura 1. Tendencia epistemológica del profesorado de biología sobre la Enseñanza de la Biología $(r>0,5)$

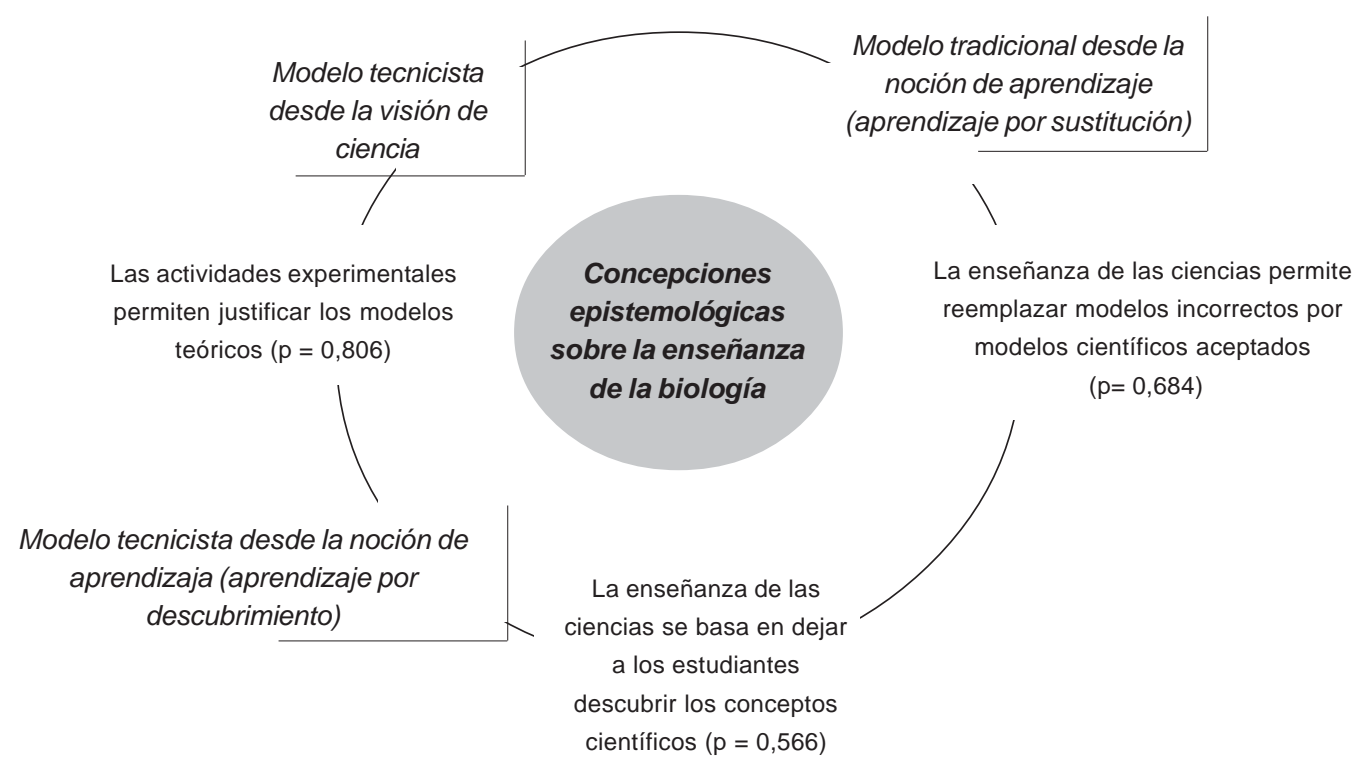

Fuente: Elaborado por los autores

a) Falta de una concepción robusta sobre las finalidades de enseñar biología para el siglo XXI. Esto conduce a un profesor lábil conceptualmente que no es capaz de sortear, adaptar, flexibilizar, contrastar e implementar un diseño de clase para el desarrollo de competencias de pensamiento científico que movilicen a los estudiantes por distintos planos de análisis (LABARRERE; QUINTANILLA, 2002) que conduzcan modos de pensar para abordar exitosamente una tarea escolar.

b) Políticas Educativas, a nivel de las bases, limitadas exclusivamente a las propuestas ministeriales y a las exigencias de mercado condicionadas por las pruebas nacionales e internacionales que caracterizan y sitúan a un colegio en un lugar o en otro.

\section{Discusión}

La enseñanza de la biología debe permitir a los jovenes interpretar y reconstruir el mundo que les ha tocado vivir, para ello, debemos reorientar sus propósitos y responder a las necesidades de los jovenes que viven y deberán enfrenta el siglo XXI; lo anterior nos obliga a transitar hacia espacios diversos y flexibles para que puedan configurarse las características individuales de nuestros jovenes y a la vez brindar posibilidades de acceso a los saberes que nuestra cultura ha configurado en el tiempo; que cambia y evoluciona según las intuiciones y valores de las personas que la construyen (IZQUIERDO, 2007) de manera que los estudiantes 
puedan integrarla a sus propias vidas para la participación consciente y toma de decisiones en nuestra sociedad; para ello,es necesario nuevos modos de pensar; promover competencias de pensamiento científico. Sin embargo, tales pretensiones se invisibilizan en el profesorado de biología por la persistente visión tradicional vinculada a nociones de aprendizaje, motivo de la enseñanza; como a los métodos que implican su enseñanza, entendida por los profesores participantes como: la enseñanza de la ciencia debe considerar las actividades experimentales ya que éstas, permiten justificar los modelos teóricos que se enseñan; ahora bien, si los modelos teóricos si son incorrectos, la enseñanza - biología - permite reemplazarlos por modelos cientificamente aceptados; el fundamento de la enseñanza en la escuela es que los estudiantes descubran los conceptos científicos. La discusión que hemos sostenido nos lleva a afirmar que el profesorado de biología deja entre ver concepciones y creencias propias sobre la enseñanza de la biología, en un marco de restricciones que se articulan como procesos para la acción pedagógica, aspecto interesante que aún estamos investigando.

Los resultados nos permiten afirmar que la enseñanza de la biología tiene una imagen tradicional, tecnicista, dogmática y positivista con un deficiente enfasis a las dimensiones sociales, valóricas, culturales y creativas de la ciencia. Desde esta perspectiva creemos que un obstáculo hacia una actividad científica escolar auténtica (RAVANAL; QUINTANILLA, 2008) es el valor asignado a las actividades experimentales y al trabajo de laboratorio; actividades con un rol motivador, verificador, cargado de procedimientos, aspecto importante y relevante para aprender según el profesorado. Los resultados permiten afirmar que la enseñanza de la biología es de caracter tradicional, tecnicista y dogmática con un deficiente enfasis en atender las dimensiones sociales, valóricas, culturales y creativas de la ciencia. Dichas desatenciones, un obstáculo para la promoción de una actividad científica escolar auténtica que contribuya al aprendizaje con sentido (RAVANAL; QUINTANILLA, 2008). El valor que asigna el profesorado a las actividades experimentales y al trabajo de laboratorio, así como su rol, en sí mismo, motivador, verificador y cargado de procedimientos, son un aspecto importante y relevante para el aprendizaje de la ciencia escolar según el imaginario docente.

En la configuración de un nuevo marco educativo global, habría que dar un paso adelante en la perspectiva de superar la dependencia de la formación, la enseñanza y el aprendizaje respecto de los hábitos y modelos “clásicamente académicos". La actividad que el estudiante desarrolla lo hace consciente de sus errores y estos se transforman en un vínculo para acceder y re-construir un conocimiento científico de mayor complejidad, evitando que en un primer momento, lo que parece comprendido e integrado, sea olvidado, dejando resurgir las representaciones que se creían superadas. A menudo, es difícil para el profesor profundizar en las ideas que tienen los alumnos además, habitualmente se dedica poco tiempo a interpretar el significado que tiene para él o para ella una afirmación inesperada que surge en un intercambio de ideas, por eso se recomienda recurrir a los instrumentos que se han diseñado desde la investigación en didáctica de las ciencias y desarrollar competencias y habilidades para la interacción social y asegurar así la regulación de los aprendizajes en un marco estratégicoevaluativo más amplio y significativo para el estudiante que aprende biología. Considerando los hallazgos de esta investigación, se hace imperativa la proposición de acciones educativas que permitan la evolución teórica de los profesores, la práctica escolar y la enseñanza de los conceptos científicos. El desarrollo de competencias de pensamiento científico permitirá, que no solamente, el profesorado asigne otro sentido a la práctica científica, sino que también, a 
sus aprendices. Así, la transmisión de contenidos no puede ser el centro de la actividad científica escolar, debe ser una actividad dinámica donde los estudiantes construyen teorías propias para interpretar y explicar el mundo en que viven. Esta mirada asume el conocimiento científico desde una visión racional instrumental, relativa y naturalista (GIERE, 1992), donde el sujeto construye y reconstruye sus conocimientos de acuerdo a sus capacidades, contexto social, sus interacciones y las dudas a las cuales tiene interés en esclarecer.

\section{Implicancias didácticas}

Abiertamente el profesorado declara una visión racionalista, empirista y positivista sobre la enseñanza de la biología, en la que su interés no está centrado en cómo se construye el conocimiento sino en justificarlo. Ahora bien, el estudio interpretativo con estudio de caso que hemos desarrollado a partir de los mapas cogntivos (RAVANAL, 2010) reafirman la idea de justificar el conocimiento desde la visión tecnicista de la enseñanza; como también revela contradicciones teóricas sobre el modo de justificarlo y su componente ideológico.

Creemos que la enseñanza de la biología no es fundamentada; no considera un análisis del contenido ha enseñar y de las dificultades de su aprendizaje. Estas restricciones u omisiones puede ser explicada por la escasa implicación del profesorado en procesos de reflexión sobre el conocimiento científico (SÁNCHEZ; VALCÁRCEL, 2002) y el conocimiento didáctico del contenido biológico. Estos aspecto llevan a una enseñanza aproblémica, orientada principalmente desde la propuesta ministerial con propósitos que enfatizan la comprensión sólida de los conceptos científicos (CONTRERAS, 2009). La enseñanza de la biología comparte un modelo de enseñanza tradicional - tecnicista con una teoría de aprendizaje dado por apropiación o asimilación de conceptos o sustitución (PORLÁN; MARTÍN DEL POZO, 2004) y el contenido disciplinar propuesto es simplificado o adaptado a partir de un método de transmisión verbal preferentemente o de secuencia de actividades cerradas que difícilmente se aproximan a las finalidades de la ciencia hoy como a las necesidades y exigencias de nuestros jóvenes para el siglo XXI.

\section{Referencias}

ACEVEDO, J. Investigación científica, naturaleza de la ciencia y enseñanza de las ciencias (II). Revista Eureka sobre Enseñanza y Divulgación de las Ciencias, Cádiz, v. 4, n. 3, p. 571-579, 2007.

ADÚRIZ-BRAVO, A. ¿Qué naturaleza de la ciencia hemos de saber los profesores de ciencias?: una cuestión actual de la investigación didáctica. [S. 1.]: Organización de las Naciones Unidas para la Educación, la Ciencia y la Cultura, 2007. Disponible en: <http:// bibliotecavirtual.educared.org/index.php/site/default/detalle/id/00000000013/quenaturaleza-de-la-ciencia-hemos-de-saber-los-profesores-de-ciencias $>$. Acceso en: 1 mar. 2011. 
Ravanal, E. M.; Quintanilla, M. G.; Labarrere, A. S.

ADÚRIZ-BRAVO, A. Integración de la epistemología en la formación del profesorado de ciencias. 2001. 622 f. Tesis (Doctorado en Didàctica de le Ciències Experimentals) - Facultad de Ciències de l'Educació, Universitat Autònoma de Barcelona, Bellaterra, 2001. Disponible en: <http://www.tdx.cat/handle/10803/4695>. Acceso en: 20 dic. 2011.

ALIBERAS, J. ¿Qué conocimiento científico enseñar en la escuela obligatoria? Enseñanza de las Ciencias, Barcelona, p. 1-5, 2005. Número extra.

ANGULO DELGADO, F. Aprender a enseñar ciencias: análisis de una propuesta para la formación inicial del profesorado de secundaria, basada en la metacognición. 2002. $529 \mathrm{f}$. Tesis (Doctorado en Didàctica de le Ciències Experimentals) - Facultad de Ciències de l'Educació, Universitat Autònoma de Barcelona, Bellaterra, 2002. Disponible en: <http:// www.tesisenred.net/handle/10803/4693 >. Acceso en: 16 ago. 2011.

CAMACHO, J.; QUINTANILLA, M. Resolución de problemas científicos desde la historia de la ciencia: retos y desafíos para promover competencias cognitivo lingüísticas en la química escolar. Ciência \& Educação, Bauru, v. 14, n. 2, p. 197-212, 2008.

CARVAJAL, E.; GÓMEZ, M. R. Concepciones y representaciones de los maestros de secundaria y bachillerato sobre la naturaleza, el aprendizaje y la enseñanza de las ciencias.

Revista Mexicana de Investigación Educativa, México, v. 7, n. 16, p. 577-602, 2002.

CHAMIZO, A.; IZQUIERDO, M. Evaluación de las competencias de pensamiento científico. Alambique: Didáctica de las Ciencias Experimentales, Barcelona, n. 51, p. 9-19, 2007.

CONTRERAS, S. Creencias curriculares y creencias de actuación curricular de los profesores de ciencias chilenos. Revista Electrónica de Enseñanza de las Ciencias, Vigo, v. 8, n. 2, p. 505-526, 2009.

EICK, C. J. Inquiry, nature of science, and evolution: the need for a more complex pedagogical content knowledge in science teaching. Electronic Journal of Science Education, Georgetown, v. 4, n. 3, 2000. Disponible en: < http://ejse.southwestern.edu/ article/view/7633>. Acceso en:10 mar. 2010.

FLORES, F. et al. Concepciones sobre la naturaleza de la ciencia de los profesores de biología de nivel secundario. Revista Mexicana de Investigación Educativa, México, v. 12, n. 32, p. 359-380, 2007.

GIERE, R. La explicación de la ciencia: un acercamiento cognoscitivo. México: Consejo Nacional de Ciencia y Tecnología, 1992.

GONZÁLEZ, A. et al. Resolver problemas para aprender: una propuesta para el desarrollo de competencias de pensamiento científico en la facultad de ciencias. Enseñanza de las Ciencias, Barcelona, p.1-5, 2005. Número extra. 
Concepciones epistemológicas del profesorado ...

GUISÁSOLA, J.; MORENTIN, M. Comprenden la naturaleza de la ciencia los futuros maestros y maestras de educación primaria? Revista Electrónica de Enseñanza de las Ciencias, Vigo, v. 6, n. 2, p. 246-262, 2007.

IZQUIERDO, M. Enseñar ciencias, una nueva ciencia. Enseñanza de las Ciencias Sociales, Barcelona, v. 6, 125-138, 2007.

. Fundamentos epistemológicos. In: PERALES, F.; CAÑAL, P. (Comp.). Didáctica de las ciencias experimentales: teoría y práctica de la enseñanza de las ciencias. Alcoy: Marfil, 2000. p. 35-64.

. Hacia una teoría de los contenidos escolares. Enseñanza de las Ciencias, Barcelona, v. 23, n. 1, p. 111-122, 2005.

IZQUIERDO, M.; SANMARTÍ, N.; ESPINET, M. Fundamentación y diseño de las prácticas escolares de ciencias experimentales. Enseñanza de las Ciencias, Barcelona, v. 17 , n. 1, p. $45-59,1999$.

LABARRERE, A. Aspectos de carácter teórico como contribución al análisis y debate: documento de trabajo para sesiones de análisis de información. Santiago de Chile: Pontificia Universidad Católica de Chile, 2008. (Proyecto Fondecyt 1070795).

La solución de problemas, eje del desarrollo del pensamiento y las competencias de pensamiento científico matemáticas y ciencias experimentales. In: QUINTANILLA, M. (Comp.). Las competencias de pensamiento científico desde "las voces" del aula: historia de un proyecto de formación continua de docentes basado en la investigación en didáctica de las ciencias. Santiago de Chile: Pontifícia Universidad Católica, 2012. v. 1. p. 4782.

LABARRERE, A.; QUINTANILLA, M. La solución de problemas científicos en el aula: reflexiones desde los planos de análisis y desarrollo. Pensamiento Educativo, Santiago de Chile, v. 30, n. 1, p. 121-137, 2002.

LEDERMAN, N. Student's and teachers'conceptions of the nature of science: a review of the research. Journal of Research in Science Teaching, Hoboken, v. 29, n. 4, p. 173-184, 1992.

'Teachers' understanding of the nature of science and classroom practice: factors that facilitate or impede the relationship. Journal of Research in Science Teaching, New York, v. 36, n.8, p. 916-929, 1999.

MARTÍNEZ, M.; MARTÍN DEL POZO, R.; RODRIGO, M. ¿Qué pensamiento profesional y curricular tienen los futuros profesores de ciencias de secundaria? Enseñanza de las Ciencias, Barcelona, v. 19, n. 1, p. 67-87, 2001.

MELLADO, V. Concepciones y prácticas de aula de profesores de ciencias, en formación inicial de primaria y secundaria. Enseñanza de las Ciencias, Barcelona, v. 14, n. 3, p. 289302, 1996. 
Ravanal, E. M.; Quintanilla, M. G.; Labarrere, A. S.

ORGANIZACIÓN PARA LA COOPERACIÓN Y EL DESARROLLO ECONÓMICO.

Informe PISA 2006: competencias científicas para el mundo del mañana. Madrid:

Santillana, 2008. Disponible en: < http://www.oecdbookshop.org/oecd/

display.asp?lang $=\mathrm{EN \& sf1}=$ identifiers\&st $1=982007014 \mathrm{e} 1>$. Acceso en: 02 oct. 2011.

PORLÁN, R.; MARTÍN DEL POZO, R. The conceptions of in-service and prospective primary school teachers about the teaching and learning of science. Journal of Science Teacher Education, Dordrecht, v. 15, n. 1, p. 39-62, 2004.

QUINTANILLA, M. Historia de la ciencia: aportes para la formación del profesorado. Santiago de Chile: Arrayán, 2007. v. 1.

. La ciencia en la escuela: un saber fascinante para aprender a "leer el mundo".

Pensamiento Educativo, Santiago de Chile, v. 39, n. 2, p. 177-204, 2006 a.

Identificación, caracterización y evaluación de competencias científicas desde una imagen naturalizada de la ciencia. In: QUINTANILLA, M.; ADÚRIZ-BRAVO, A. (Ed.).

Enseñar ciencias en el nuevo milenio: retos y propuestas. Santiago de Chile: Pontifícia Universidad Católica, 2006b. p. 117-142.

La investigación en evaluación de competencias de pensamiento desde la formación continua del profesorado: algunas directrices epistemológicas. In:

QUINTANILLA, M. (Comp.). Las competencias de pensamiento científico desde "las voces" del aula: historia de un proyecto de formación continua de docentes basado en la investigación en didáctica de las ciencias. Santiago de Chile: Pontifícia Universidad Católica, 2012. v. 1. p. $15-46$.

QUINTANILLA, M. et al. Identificación, caracterización y evaluación de competencias de pensamiento científico en profesores de ciencia en formación a través del enfrentamiento a la solución de problemas. Santiago de Chile: Pontificia Universidad Católica de Chile, 2011. (Proyecto Fondecyt 1110598).

QUINTANILLA, M. et al. Desarrollo, caracterización y validación de un modelo de evaluación de competencias de pensamiento científico en estudiando de enseñanza media basado en el enfrentamiento a la resolución de problemas para promover aprendizajes de calidad. Santiago de Chile: Pontificia Universidad Católica de Chile, 2009. (Proyecto Fondecyt 1095149).

QUINTANILLA, M. et al. Identificación, caracterización y promoción de competencias de pensamiento científico en estudiantes de secundaria, mediante el enfrentamiento a la resolución de problemas: un aporte a la Reforma. Santiago de Chile: Pontificia Universidad Católica de Chile, 2007. (Proyecto Fondecyt 1070795).

QUINTANILLA, M. et al. Elaboración, validación y aplicación preliminar de un cuestionario sobre ideas acerca de la imagen de ciencia y educación científica de profesores en servicio. Boletín de Investigación Educacional, Santiago de Chile, v. 21, n. 2, p. 103-132, 2006. 
Concepciones epistemológicas del profesorado ...

RAVANAL, E. Racionalidades epistemológicas y didácticas del profesorado de biología en activo sobre la enseñanza y aprendizaje del metabolismo: aportes para el debate de una nueva clase de ciencias. 2010. Tesis (Doctorado en Educación) - Universidad Academia de Humanismo Cristiano, Santiago de Chile, 2010. 1 CD-ROM.

RAVANAL, E.; QUINTANILLA, M. De las actividades curriculares científicas "tradicionales" a las actividades científicas escolares "auténticas". Aportes para el debate de una "nueva clase de ciencias". In: ENCUENTROS DE DIDÁCTICA DE LAS CIENCIAS EXPERIMENTALES, 23., Almería, 2008. Actas... Almería: Asociación Española de Profesores y Investigadores de Didáctica de las Ciencias Experimentales, 2008. p. 643-650.

RUIZ, C. et al. Construcción de mapas cognitivos a partir del cuestionario INPECIP: aplicación al estudio de la evolución de las concepciones de una profesora de secundaria entre 1993 y 2002. Revista Electrónica de Enseñanza de las Ciencias, Vigo, v. 4, n. 1, 2005.

SÁNCHEZ, G.; VALCÁRCEL, M. V. ¿Qué tienen en cuenta los profesores cuando seleccionan el contenido de enseñanza?: cambios y dificultades tras un programa de formación. Enseñanza de las Ciencias, Barcelona, v. 18, n. 3, p. 423-437, 2002.

SETTLE, T. How to avoid implying that physicalism is true: a problem for teachers of science. International Journal of Science Education, Abingdon, v. 12, n. 3, p. 258-264, 1990.

TOULMIN, S. La comprensión humana. Madrid: Alianza Universidad, 1977.

WANG, A. H.; MARSH, D. D. Science instruction with a humanistic twist: teachers' perception and practice in using the history of science in their classrooms. Science $\boldsymbol{\&}$ Education, Dordrecht, v. 11, n. 2, p. 169-189, 2002. 